\title{
EMBEDDING RINGS WITH A MAXIMAL CONE AND RINGS WITH AN INVOLUTION IN QUATERNION ALGEBRAS
}

\author{
BY
}

\author{
CARL W. KOHLS AND WILLIAM H. REYNOLDS
}

\begin{abstract}
Sufficient conditions are given for an algebra over a totally ordered field $F$ to be isomorphic to a subring of the algebra of quaternions over the real closure of $F$. These conditions include either the requirement that the nonnegative scalars form a maximal cone in the algebra, or that the algebra have an involution such that the scalars are the only symmetric elements. For many matrix algebras, the cone requirement alone is imposed.
\end{abstract}

1. Introduction. In [5], it was shown that the presence of a certain type of maximal cone in a commutative ring ensures that it is isomorphic to a subring of the complex numbers, and under certain restrictions any ring with such a cone is embeddable in the real quaternions. The present investigation began as an attempt to determine whether the restrictions could be removed in rings of real matrices. The general question is still open, but we have obtained an embedding for a large class of matrix rings, assuming the existence of a maximal cone that is restricted only with respect to its position in the ring. This depends upon a result about algebras with an involution, a special case of which is the following: A selfadjoint algebra of operators on a real inner product space in which the only symmetric elements are the scalars is isomorphic to a subring of the real quaternions. The general theorem does not assume a norm, however. Other embedding results, involving a maximal cone or the existence of elements satisfying a quadratic equation, are obtained from the same basic lemmas.

The proofs of some of the lemmas are variations on the proof by R. S. Palais of the classical Frobenius theorem on real division algebras [4]. A vital fact in the proof of that theorem is that the complex field is algebraically closed; this of course is equivalent to the $\mathrm{f}$ act that the real field is real closed. We work with algebras over any totally ordered field, and simply use the fact that such a field has a real closure.

2. Preliminaries. A (positive) cone in a ring $A$ is a subsemiring $P$ containing 0 such that if $a \in P$ and $-a \in P$, then $a=0$. If $A$ is a partially ordered ring, then $\{a \in A: a \geq 0\}$ is a cone; and if $P$ is a cone in a ring $A$, then $A$ is a

Presented to the Society, January 28, 1973; received by the editors July 1, 1971. AMS (MOS) subject classifications (1970). Primary 06A70, 16A28, 46K99; Secondary $16 \mathrm{~A} 42$. 
partially ordered rin; with the order defined by $a \geq b$ if and only if $a-b \in P$. A maximal cone in $A$ is a cone that is maximal in the family of cones in $A$ (ordered by set inclusion). By Zorn's lemma, every cone is contained in a maximal cone. Any cone $P$ in $A$ corresponding to a total order (so that either $a \in P$ or $-a \in P$ for all $a \in A$ ) is a maximal cone. If a maximal cone $M$ in a ring $A$ with identity 1 is contained in the center of $A$ and $1 \in M$, then for any $a \notin M$, there is a finite sequence $m_{0}, m_{1}, \cdots, m_{n}$ of elements in $M$, not all zero, such that $m_{0}+$ $\sum_{j=1}^{n} m_{j} a^{j}=0$.

Recall that a real closed field is a field that is maximal in the family of totally ordered algebraic extensions of a totally ordered field (ordered by set inclusion). A field $R$ is real closed if and only if $R(\sqrt{-1})$ is algebraically closed, which occurs if and only if the following two conditions hold:

(i) every positive element in $R$ has a square root in $R$;

(ii) every polynomial of odd degree over $R$ has a root in $R$.

A demiring in a ring $A$ with identity 1 is a subsemiring $D$ of $A$ such that $1 \in D$ and $-1 \notin D$. A bemiring is a maximal demiring. A conic demiring is a demiring that is also a cone. We observe that every conic hemiring is a maximal cone. But the converse is false. For example, let $R$ be the real field with the usual order and let $A=R \oplus R$. It is easy to see that $\{(r, s) \in A: r \geq 0, s \geq 0\}$ is a maximal cone in $A$. But $\{(r, s) \in A: s \geq 0\}$ is a demiring that contains it properly, so it is not a hemiring.

D. K. Harrison has proved the following statement [1, Corollary 1.8]. Let $A$ be a ring with identity 1 . If $A$ contains a conic hemiring $H$ with the properties

(B) for each $b \in H$ there is a positive integer $n$ such that $n 1-b \in H$, and

(G) $A=H-H$, then there exists a monomorphism of $A$ into the real field taking $H$ to the nonnegative numbers in the image of $A$.

The nonnegative real numbers in the complex field or in the algebra of real quaternions also form a conic hemiring $H$, but having only property (B). (See, e.g., [5, Theorem 3] for proof.) The first author conjectured that if a ring $A$ with identity contains a conic hemiring $H$ with property (B), then there ex ists a monomorphism of $A$ into the algebra of real quaternions taking $H$ to the nonnegative real numbers in the image of $A$. (Presumably one would be able to characterize when the image is contained in the real or complex fields.) In [5], the second author used Harrison's theorem on the subring $H-H$ to prove the following results. If $A$ is commutative, there is such a monomorphism into the complex field. If $H$ is is omorphic to the nonnegative real numbers and if

(N) for each $h \in H$ and $a \in A$, there exists $b^{\prime} \in H$ such that $a b=b^{\prime} a$, 
then there is such a monomorphism into the real quaternions.

If $(\mathrm{N})$ holds, there is always a monomorphism into a division algebra that is algebraic over a subfield of the real field.

The present paper began as an attempt to prove the conjecture for matrix rings over the real field. However, we were unable to exploit Harrison's result, and so changed the hypotheses by postulating only the existence of a maximal cone in a ring of $n \times n$ matrices over any real closed field, but requiring that this cone be the set of nonnegative scalar matrices in the ring. With the additional requirement that the ring be closed under transposition when $n$ is even and $n>2$, we obtain the desired monomorphism. The investigation led to several other results, not always involving a maximal cone.

Let $F$ denote a totally ordered field, $R$ its real closure, $C$ its algebraic closure (so that $C=R(\sqrt{-1})$ ), and $Q$ the algebra of quaternions over $R$. (In Lemmas 9 and 10 and in Theorem 3, $R$ simply means any real closed field.) Given an $F$-algebra $A$ with identity, there is no loss of generality in assuming that $A$ is a subring of an $R$-algebra with the same identity, since, if $A$ is identified with an isomorphic copy, $A \otimes_{F} R$ is such an algebra. Let 1 be the identity of $A$ and $r \in R$; we shall write $r$ for $r 1$, and $F$ for $\{r 1 \in A: r \in F\}$. For any $x \in A$, let $C(x)=C_{A}(x)=\{y \in A: y x=x y\}$ and $C^{-}(x)=C_{A}^{-}(x)=\{y \in A: y x=-x y\}$. Note that $C(x)$ and $C^{-}(x)$ are subspaces of the $F$-space $A$. We denote the subalgebra of $A$ generated by 1 and $x$ by $F[x]$.

An $F$-algebra $A$ with identity that has an involution, i.e., a nonsingular linear transformation $a \longmapsto a^{*}$ of period 2 such that $(a b)^{*}=b^{*} a^{*}$ for all $a, b \in A$, will be called an $F^{*}$-algebra. An element $a$ of an $F^{*}$-algebra is symmetric if $a^{*}=a$ and skew if $a^{*}=-a$. Note that the scalars are symmetric elements, as are elements of the form $a a^{*}$.

The symbol $R_{n}$ denotes the $R$-algebra of all $n \times n$ matrices over $R$. A subring of $R_{n}$ that contains the transpose of each of its elements will be called selfadjoint. Note that any $n$-dimensional $R$-space has an inner product, so that any subring of $R_{n}$ is a ring of operators on an $n$-dimensional $R$-space with an inner product.

3. Maximal cones and quadratic polynomials. In this section, $A$ always denotes an $F$-algebra with identity. The basic lemmas are proved here, and some applications made. Further applications will be given in the last section.

Lemma 1. Let $x \in A$ satisfy an irreducible quadratic polynomial over $F$, and let $a \in F[x]$, with $a \notin F$. Assume that $b \in C(x)$, and

(1) both $b$ and $a b$ satisfy quadratic polynomials over $F$. Then $b \in F[x]$. 
Proof. Suppose not. Note that $b \in C(a)$, and that $F[x]$ is a field, whence $a$ is a unit in $F[x]$. Since $b \notin F, b$ does not satisfy a linear polynomial over $F$; neither does $a b$, because $F\left[a^{-1}\right] \subseteq F[x]$ and $b \notin F[x]$. Hence for some $r, s, t$, $u \in F$, we have $a^{2} b^{2}+r a b+s=0$ and $b^{2}+t b+u=0$. Then $a^{2}(-t b-u)+r a b+$ $s=0$, so that $(r-t a) b \in F[x]$. Since $a \notin F, r-t a$ is a unit in $F[x]$, and thus $b \in F[x]$, a contradiction.

Lemma 2. Let $x \in A$ satisfy $x^{2}+t=0$, where $t \in F$ and $t \neq 0$. Then $A$ is the direct sum of the F-spaces $C(x)$ and $C^{-}(x)$.

Proof. For any $a \in A, 1 / 2\left(a-t^{-1} x a x\right) \in C(x), 1 / 2\left(a+t^{-1} x a x\right) \in C^{-}(x)$, and $a=1 / 2\left(a-t^{-1} x a x\right)+1 / 2\left(a+t^{-1} x a x\right)$. Thus $A=C(x)+C^{-}(x)$. If $b \in C(x) \cap C^{-}(x)$, then $x b=-x b$, so that $2 t b=-2 x^{2} b=0$, whence $b=0$.

Lemma 3. Let $z \in A$ satisfy $z^{2}+s=0$, where $s \in F$ and $s \neq 0$. Assume that $C(z)=F[z]$. If $y \in C^{-}(z)$, then $y^{2}+t=0$ for some $t \in F$.

Proof. Clearly we may assume that $y \neq 0$. Since $y^{2} \in C(z)=F[z]$, and $z^{2}=$ $-s$, we have $y^{2}=-t+r z$, where $r, t \in F$. Thus $y(-t+r z)=(-t+r z) y$, whence $2 r z y=0$, and $2 r s y=-2 r z^{2} y=0$. It follows that $r=0$, so that $y^{2}+t=0$.

Note that Lemma 1 remains valid if $F$ is replaced by any field, and Lemmas 2 and 3 remain valid if $F$ is replaced by any field with characteristic different from 2 .

Lemma 4. Let $x \in A$ satisfy $x^{2}+s=0$, where $s \in F$ and $s>0$. Assume that

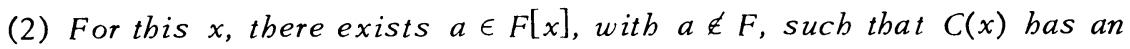
$F$-basis of elements $b$ satisfying (1).

Then $C(x)=F[x]$. Furthermore, if $A$ is commutative, then it is F-isomorpbic to a subfield of $C$; and if there exists $y \in C^{-}(x)$ such that $y^{2}+t=0$, where $t \in F$ and $t>0$, then $A$ is $F$-isomorphic to an F-subalgebra of $Q$.

Proof. Since $s>0, x$ satisfies an irreducible quadratic polynomial over $F$, and $F[x]$ is $F$-isomorphic to a subfield of $C$. By Lemma $1, F[x]$ contains an $F$-basis of $C(x)$, whence $F[x]=C(x)$. Thus, if $A$ is commutative, we have $A=$ $C(x)$, and $A$ is $F$-isomorphic to a subfield of $C$.

Now assume there exists $y \in C^{-}(x)$ such that $y^{2}+t=0$, where $t \in F$ and $t>0$. It is easily seen that right multiplication by $y$ is a nonsingular linear transformation mapping $C(x)$ onto $C^{-}(x)$. But $\{1, x\}$ is an $F$-basis for $C(x)$, so that $\{y, x y\}$ is an $F$-basis for $C^{-}(x)$. Consequently, from Lemma $2,\{1, x, y, x y\}$ is an $F$-basis for $A$. Since $(x y)^{2}+s t=0$, where $s t>0$, and $x y \in C^{-}(x) \cap C^{-}(y)$, it follows that the mapping $r_{1}+r_{2} x+r_{3} y+r_{4} x y ! \rightarrow r_{1}+r_{2} \sqrt{s} i+r_{3} \sqrt{t} j+r_{4} \sqrt{s t} k$ is a monomorphism of $A$ into $Q$. 
Lemma 5. Let $M=\{r \in F: r \geq 0\}$ be a maximal cone in $A$, and let $\bar{A}$ be an $R$-algebra containing $A$ with the same identity. Then $\bar{A}$ bas no left zero divisor of the form $a-r$, where $a \in A$ and $r \in R$.

Proof. Let $a \in A$ and $r \in R$. If $a \in F$, it is clear that $a-r$ cannot be a left zero divisor in $\bar{A}$. Assume that $a \notin F$, and $(a-r) x=0$ for some $x \in \bar{A}$. Since $-a \notin F$ and $(a+1) \notin F$, we may assume that $r>0$. Maximality of $M$ implies that for some sequence $m_{0}, \cdots, m_{n}$ of elements in $M$, not all zero, we have $m_{0}{ }^{+}$ $\sum_{j=1}^{n} m_{j} a^{j}=0$. Now $a x=r x$, so that $\left(m_{0}+\sum_{j=1}^{n} m_{j} r^{j}\right)_{x}=\left(m_{0}+\sum_{j=1}^{n} m_{j} a^{j}\right)_{x=0}$. Since $m_{0}+\sum_{j=1}^{n} m_{j} r^{j}>0$, we have $x=0$.

Lemma 6. Let $\{r \in F: r \geq 0\}$ be a maximal cone in $A$. Then $A$ is a division algebra that is algebraic over $F$.

Proof. It suffices to consider $a \notin F$. Then for some sequence $r_{0}, \cdots, r_{n}$ of elements in $F$, not all zero, we have $\sum_{j=1}^{n} r_{j} a^{j}+r_{0}=0$. Since $a$ is not a left zero divisor, by Lemma 5, we may assume that $r_{0} \neq 0$. Thus $a$ is a unit, with $a^{-1}=-r_{0}^{-1}\left(\sum_{j=2}^{n} r_{j} a^{j-1}+r_{1}\right)$.

Lemma 7. Let $M=\{r \in F: r \geq 0\}$ be a maximal cone in $A$, and let $z \in A$, with $z \notin F$. If $z^{2}+s=0$, for some $s \in F$, then $s>0$.

Proof. By Lemma 6, $A$ is a division algebra, so that $s \neq 0$. Suppose $s<0$. Since $z \notin F$, we have $m_{0}+\Sigma_{j=1}^{n} m_{j} z^{j}=0$ for some sequence $m_{0}, \cdots, m_{n}$ of elements in $M$, not all zero. Replacing $z^{2}$ by $-s \in M$ in this equation, we obtain $q_{0}+q_{1} z=0$ for some $q_{0}, q_{1} \in M$, with $q_{1} \neq 0$. But then $z=-q_{1}^{-1} q_{0} \in F$, a contradiction. Thus $s>0$.

Proposition 1. Let $M=\{r \in F: r \geq 0\}$ be a maximal cone in A. Assume that there exists $x \in A$, with $x \notin F$, satisfying a quadratic polynomial over $F$, and that (2) bolds. Then $A$ is F-isomorphic to an F-subalgebra of $Q$; and if $A$ is commutative it is F-isomorphic to a subfield of $C$.

Proof. Since $x \notin F$, it satisfies a monic quadratic polynomial over $F$, whence $(x+r)^{2}+s=0$ for some $r, s \in F$. Let $z=x+r$. Since $z \notin F$, we have $s>0$ by Lemma 7. Thus $F[z]=F[x], C(z)=C(x)$, and $z^{2}+s=0$ with $s>0$, so that $z$ satisfies the hypotheses of Lemma 4; hence $C(z)=F[z]$. If $A$ is not commutative, then, since $C(z)$ is commutative, $A \neq C(z)$. By Lemma 2, there exists a nonzero $y \in C^{-}(z)$. From Lemma $3, y^{2}+t=0$, where $t \in F$. Since $y \notin F$, we have $t>0$ by Lemma 7. Lemma 4 now implies the conclusions.

Lemma 8. Let $\bar{A}=A \otimes_{F} R$. If $a \in A$ and $x=r_{1} a+r_{2}$, where $r_{1}, r_{2} \in R$ and $r_{1} \neq 0$, then the set $C_{A}(a)$ is an R-generating set for $C_{\bar{A}}(x)$. 
Proof. Let $y \in C_{\bar{A}}(x)$, and write $y=\sum_{j=1}^{n} a_{j} \otimes s_{j}$, where $s_{1}, \cdots, s_{n} \in R$ are linearly independent over $F$, and $a_{1}, \cdots, a_{n} \in A$. Since $y \in C_{\bar{A}}(a)$, we have $\sum_{j=1}^{n}\left(a a_{j}\right) \otimes s_{j}=\sum_{j=1}^{n}\left(a_{j} a\right) \otimes s_{j}$, so that $\sum_{j=1}^{n}\left(a a_{j}-a_{j} a\right) \otimes s_{j}=0$, whence $a a_{j}-a_{j} a=0(j=1, \cdots, n)\left[3\right.$, p. 21]. Thus, $a_{1}, \cdots, a_{n} \in C_{A}(a)$.

Proposition 2. Let $\bar{A}=A \otimes_{F} R$. Assume that there exists $a \in A$, with $a \notin F$, such that $(a+r)^{2} \in R$ and $(a+r)^{2}<0$ for some $r \in R$, and that each element of $C_{A}(a)$ satisfies a quadratic polynomial over $R$. If $A$ is commutative, then $\bar{A}$ is isomorphic to $C$. If $A$ is noncommutative and $\bar{A}$ bas no nonzero nilpotent elements, then $\bar{A}$ is isomorphic to $Q$.

Proof. Let $(a+r)^{2}+s=0$, where $s \in R$ and $s>0$; set $x=a+r$, so that $x^{2}+s=0$. Clearly $a \in R[x]$ and $a \notin R$. By Lemma $8, C_{A}(a)$ is an R-generating set for $C_{\bar{A}}(x)$; and the elements of $C_{A}(a)$ satisfy (1) with $F=R$. From Lemma 4, $C_{\bar{A}}(x)=R[x]$. Now $\bar{A}$ is commutative if and only if $A$ is commutative; so if $A$ is noncommutative, then $\bar{A} \neq C_{\bar{A}}(x)$, and by Lemma 2 there is a nonzero $y$ in $C_{\bar{A}}(x)$. From Lemma $3, y^{2}+t=0$ for some $t \in R$, and here we have $t \neq 0$. If $t<0$, then there are nonzero $q_{1}, q_{2} \in R$ such that $-t=q_{1}^{2}$ and $s=q_{2}^{2}$, whence $\left(q_{1} x+q_{2} y\right)^{2}$ $=-t x^{2}+s y^{2}=0$; but $q_{1} x+q_{2} y \neq 0$, by Lemma 2 , a contradiction. Hence $t>0$. Lemma 4 now gives the monomorphisms, and from its proof it is clear that they are epimorphisms.

Theorem 1. Let $\bar{A}=A \otimes_{F} R$, and let $M=\{r \in F: r \geq 0\}$ be a maximal cone in A. Assume that there exists $a \in A$, with $a \notin F$, such that $\bar{A}$ bas no zero divisor of the form $b^{2}+s b+t$, where $b \in C_{A}(a)$, and $s, t \in R$. If $A$ is commutative, then $\bar{A}$ is isomorpbic to $C$. If $A$ is noncommutative and $\bar{A}$ bas no nonzero nilpotent elements, then $\bar{A}$ is isomorphic to $Q$.

Proof. By Lemmas 5 and $6, A$ is algebraic over $F$ and $\bar{A}$ has no left zero divisor of the form $a-q$, where $a \in A$ and $q \in R$. If $b \in C_{A}(a)$, then $b$ satisfies a polynomial over $R$, which factors over $R$ into quadratic and linear polynomials. It follows that $b$ satisfies a quadratic polynomial over $R$. In particular, we have $(a+r)^{2}+s=0$ for some $r, s \in R$. If $s \leq 0$, then there exists $t \in R$ such that $-s=t^{2}$, whence $(a+r-t)(a+r+t)=0$; but the maximality of $M$ implies that $A \cap R=F$, so that $a+r-t \neq 0$ and $a+r+t \neq 0$, which is impossible in $\bar{A}$. Hence $s>0$. The conclusions now follow from Proposition 2 .

4. Algebras with an involution and matrix rings. In this section we obtain further embeddings, using the same tools, but starting with somewhat different hypotheses. We assume throughout that the ring $A$ has an identity.

Theorem 2. Let $A$ be an $F^{*}$-algebra such that the only symmetric elements are the scalars. Assume that for each nonzero skew $a \in A$, we bave $a a^{*}>0$ in $F$. 
Then there exists an F-algebra monomorphism $\phi$ of $A$ into $Q$ such that $\phi(A)=R$ if and only if $\Lambda-F$, and $\phi(\Lambda) \subseteq C$ if and only if $\Lambda$ is commutative.

Proof. Each $a \in A$ is the sum of the scalar $\left(a+a^{*}\right) / 2$ and the skew element $\left(a-a^{*}\right) / 2$. If 0 is the only skew element of $\Lambda$, then $\Lambda-F$, so that $\phi$ exists and $\phi(\Lambda) \subseteq R$. We now assume that some skew $x \in A$ is nonzero. Then $x x^{*}=s$ for a positive $s \in F$, so that $x^{2}+s=0$. For $b \in C(x)$, write $b=r+d$, where $r \in F$ and $d \in A$ is skew. Now $d^{2}+q=0$ for some $q \epsilon F$, so that $b^{2}-2 r b+r^{2}+q=0$. Thus, (2) holds with $a=x$.

We now assume that there exists a nonzero $y \in C^{-}(x)$. Since $x$ is skew, it is easy to see that $y^{*} \in C^{-}(x)$, whence $y-y^{*} \in C^{-}(x)$; and $y-y^{*} \neq 0$, for otherwise $y$ would be a scalar, which is impossible. So we may assume that $y$ is skew. As before $y^{2}+t=0$, where $t \in F$ and $t>0$. The existence of a monomorphism $\phi$ such that $\phi(A) \subseteq C$ if $A$ is commutative now follows from Lemma 4.

It is obvious that $\phi(A) \subseteq C$ implies that $\Lambda$ is commutative. Furthermore, it is clear from the proof of Lemma 4 that if some skew element of $\Lambda$ is nonzero, then $\phi(A) \not R$. Hence $\phi(A) \subseteq R$ implies that the only skew element is zero, whence $\Lambda=F$.

Corollary. Let $A$ be an $F^{*}$-algebra of linear operators on an $R$-space $V$ with an inner product $(\cdot,$.$) , such that for v, w \in V$ and $b \in \Lambda$, we have $(b v, w)=$ $\left(v, b^{*} w\right)$. As sume that the only symmetric elements are the scalars. Then there exists an F-algebra monomorphism $\phi$ of $A$ into $Q$ such that $\phi(A) \subseteq R$ if and only if $A-F$, and $\phi(A) \subseteq C$ if and only if $A$ is commutative. If, in addition, $n=$ $\operatorname{dim} V<\infty$, then $\phi(\Lambda) \subseteq R$ if $n$ is odd, and $\phi(\Lambda) \subseteq C$ if $n=2$.

Proof. Let $a \in A$ be a nonzero skew element, and choose $v \in V$ such that $a v \neq 0$. If $a a^{*}=r \in F$, then $0<\left\|a^{*} v\right\|^{2}=\left(a^{*} v, a^{*} v\right)=\left(a a^{*} v, v\right)-r(v, v)=r\|v\|^{2}$, whence $r>0$. Thus, Theorem 2 applies. Now assume that $n<\infty$. We can represent $A$ by a subring of $R_{n}$ so that the involution on $A$ corresponds to transposition in $R_{n}$.

If $n$ is odd, every skew matrix in $R_{n}$ is singular. But if $b \in A$ is skew and $b \neq 0$, then $b^{2}=q$ for some nonzero $q \in F$, and $b\left(q^{-1} b\right)=\left(q^{-1} b\right) b=1$, whence $b$ is a unit. Hence in this case 0 is the only skew element of $A$, so that, from the proof of Theorem 2, we have $\phi(A) \subseteq R$.

If $n=2$, the skew element $x$ used in the proof of Theorem 2 is represented by a skew matrix in $R_{2}$. For any $y \in C^{-}(x)$, a calculation shows that $y x=-x y$ implies that the matrix representing $y$ is symmetric with zero trace, so that $y=0$. It was shown in the proof of Theorem 2 that the hypotheses of Lemma 4 hold. Hence by Lemmas 2 and $4, A=C(x)=F[x]$. Thus $A$ is commutative, and $\phi(A) \subseteq C$. If $F=R$, the assumption in Theorem 2 that $a a^{*}>0$ for each nonzero skew 
$a \in A$ is implied by the familiar assumptions that $A$ has no nonzero nilpotent elements and that $A$ is "symmetric", i.e., that $b b^{*}+1$ is a unit for all $b \in A$. For in that case, if $a$ is a nonzero skew element, $a a^{*}=0$ implies that $a^{2}=0$, while $a a^{*}=r<0$ implies that $b b^{*}+1=0$, where $b=(\sqrt{-r})^{-1} a$.

For other results about algebras with an involution in which conditions are imposed on the symmetric elements, see the opening paragraph and the theorems in [2].

From now on, $R$ denotes an arbitrary real closed field.

Lemma 9. Let $A$ be a subring of $R_{n}$ with the same identity as $R_{n}$, such that $A \cap R$ is a field $F$ and $M=\{r \in F: r \geq 0\}$ is a maximal cone in A. If $a \in A$ and $a$ bas a characteristic root in $R$, then $a \in F$. If $n$ is odd, then $A=F$.

Proof. Let $r \in R$ be a characteristic root of $a \in A$. If $a \neq r$, then $a-r$ is a left zero divisor in $R_{n}$, which is impossible by Lemma 5 . Hence $a=r$, so that $a \in A \cap R=F$. If $n$ is odd, then every polynomial over $R$ of degree $n$ has a root in $R$, so that $a \in F$ for every $a \in A$.

Lemma 10. Let $A$ be a subring of $R_{2}$ such that every matrix in $A$ with a characteristic root in $R$ is a scalar matrix. Then there exists a scalar-preserving monomorphism $\phi$ of $A$ into $C$ sucb that $\phi(A) \subseteq R$ if and only if $A \subseteq R$.

Proof. If every element of $A$ has a characteristic root in $R$, then $A \subseteq R$, and hence $A$ is isomorphic to a subring of $R$.

Otherwise, let $a \in A$ have characteristic roots $r \pm s i$, where $r, s \in R$, and $s \neq 0$. Then there is a scalar-preserving monomorphism of $A$ into $R_{2}$ taking $a$ to $\left(\begin{array}{cc}r & s \\ -s & r\end{array}\right)$, since the two matrices have the same invariant factors and hence are similar; so we may assume that $a$ is this matrix. For any

$$
b=\left(\begin{array}{ll}
t & u \\
v & w
\end{array}\right)
$$

in $A$, we find that

$$
a b-b a=s\left(\begin{array}{cc}
v+u & w-t \\
w-t & -v-u
\end{array}\right)
$$

has characteristic roots $\pm s\left((v+u)^{2}+(w-t)^{2}\right)^{1 / 2}$. Since $s \neq 0$, we have $v=-u$ and $w=t$, so that

$$
b=\left(\begin{array}{cc}
t & u \\
-u & t
\end{array}\right)
$$

The existence of a scalar-preserving monomorphism $\phi$ of $A$ into $C$ is now clear, and we have neither $A \subseteq R$ nor $\phi(A) \subseteq R$.

Theorem 3. Let $A$ be a subring of $R_{n}$ uith the same identity as $R_{n}$, and let $M=:=\{r \in A \cap R: r \geq 0\}$ be a maximal cone in A. Assume that $A$ is selfadjoint if 
$n$ is even and $n>2$. Then there exists a monomorphism $\phi$ of $A$ into $Q$ such that $\phi(A) \subseteq R$ if and only if $A \subseteq R$, and $\phi(A) \subseteq C$ if and only if $A$ is commutative. Furtbermore, $\phi(A) \subseteq R$ if $n$ is odd and $\phi(A) \subseteq C$ if $n=2$.

Proof. Let $F$ be the field of quotients of $A \cap R$. It is easy to verify that $B=\left\{r a \in R_{n}: a \in A, r \in F\right\}$ is a subring of $R_{n}$ with the same identity as $R_{n}$ such that $B \cap R=F$, that $B$ is selfadjoint if $n$ is even and $n>2$, and that $\{r \in F$ : $r \geq 0\}$ is a maximal cone in $B$. By Lemma 9, any matrix in $B$ with a characteristic root in $R$ (in particular, any symmetric matrix in $B$ ) is a scalar matrix, and if $n$ is odd, there is a monomorphism of $B$ into $R$ with the required properties.

Lemma 10 gives the result for $B$ if $n=2$, and the corollary to Theorem 2 applies if $n$ is even and $n>2$, because transposition is an automorphism making $B$ into an $F^{*}$-algebra of the required type. Since the conclusions hold for $B$, and in each case $\phi$ preserves scalars, they also hold for $A$.

\section{REFERENCES}

1. D. K. Harrison, Finite and infinite primes for rings and fields, Mem. Amer. Math. Soc. No. 68 (1966). MR $34 \# 7550$.

2. I. N. Herstein and Susan Montgomery, A note on division rings with involutions, Michigan Math. J. 18 (1971), 75-79. MR $44 \# 250$.

3. D. G. Northcott, An introduction to homological algebra, Cambridge Univ. Press, New York, 1960. MR 22 \#9523.

4. R.S. Palais, The classification of real division algebras, Amer. Math. Monthly 75 (1968), 366-368. MR 37 \#4119.

5. W. H. Reynolds, Embedding a partially ordered ring in a division algebra, Trans. Amer. Math. Soc. 158 (1971), 293-300. MR 44 \#259.

DEPARTMENT OF MATHEMATICS, SYRACUSE UNIVERSITY, SYRACUSE, NEW YORK 13210

DEPARTMENT OF MATHEMATICS, SUNY, COLLEGE AT CORTLAND, CORTLAND, NEW YORK 13045 